\title{
TDB or TCB: does it make a difference?
}

\author{
Sergei A. Klioner \\ Lohrmann Observatory, Dresden Technical University, \\ Mommsenstraße 13, D-01062 Dresden, Germany \\ email: klioner@rcs.urz.tu-dresden.de
}

\begin{abstract}
We argue that with the refined definition of TDB to be adopted by the IAU, the transformation between TDB and TCB becomes trivial.
\end{abstract}

Keywords. time, relativity, standards

Although the relativistic framework as adopted by the IAU in 1991 and refined in 2000 recommends the coordinate time TCB to be used for the modeling of any physical phenomena not localized to the vicinity of the Earth, an older time scale TDB is often used instead. In this presentation the current status of TDB, its relation to TCB as well as its advantages and disadvantages for practical problem will be discussed in detail.

It is argued that with the refined definition of TDB to be adopted by the IAU, the transformation between TDB and TCB becomes trivial. Special attention will be paid to the use of TCB and TDB for developing new solar system ephemerides. A clear algorithm to make the time argument of future ephemerides fully consistent with TCB will be presented. A few open issues, where additional relativity-related conventions are still required will also be discussed. 